\title{
EFFECT OF SILVER DIAMINE FLUORIDE PRETREATMENT ON MICROLEAKAGE AND SHEAR BOND STRENGTH OF RESIN MODIFIED GLASS IONOMER CEMENT TO PRIMAY DENTIN (IN-VITRO STUDY)
}

\author{
Nada N. Soliman ${ }^{* *} B D S$, Nevin S. Bakry' $P h D$, Mona H. Mohy El Din ${ }^{3} P H D$, Dalia M. Talaat ${ }^{4} P h D$
}

\begin{abstract}
:
OBJECTIVES: To evaluate in vitro, the effect of 38\% silver diamine fluoride (SDF) pretreatment on the microleakage and shear bond strength of resin-modified glass ionomer cement to primary dentin.

MATERIALS AND METHODS: A total of 80 extracted sound primary molars were included in this in-vitro study and randomly assigned into four equal groups ( $\mathrm{n}=20$ teeth/group) according to the evaluation test performed. For microleakage test, class $\mathrm{V}$ cavities were prepared on the buccal surface of teeth in Group I (experimental)(n=20): Primary dentin pretreated with 38\% SDF for three minutes and Group II (control)(n=20): Primary dentin treated with distilled water for three minutes. Class V cavities were restored with RMGIC, thermo-cycled, immersed into $1 \%$ methylene blue solution for 24h, sectioned longitudinally in a bucco-lingual direction and then examined under stereomicroscope at 40x magnification. Also forty teeth were assigned for SBS test and randomly grouped into Group III (experimental) (n=20): Primary dentin flat surface pretreated with 38\% SDF. Group IV (control) (n=20): Primary dentin flat surface pretreated with distilled water. Using a Universal Testing machine SBS were determines. Failure mode was evaluated using stereomicroscope. Data were collected and subjected to statistical analysis.

RESULTS: There was no statistically significant difference in microleakage between experimental and the control group (P=0.21). However, the experimental group had significantly higher SBS than the control group. $(\mathrm{P}=0.0001)$.

CONCLUSIONS: Primary dentin pretreatment with 38\% SDF does not influence the marginal seal of RMGIC to primary dentin. However, it raises the shear bond strength between them.

KEYWORDS: Resin modified glass ionomer cement, Silver diamine fluoride, Microleakage, Shear bond strength.

RUNNING TITTLE: Effect of SDF on adhesion of RMGIC to primary dentin.
\end{abstract}

1. Bachelor of Dentistry, Faculty of Dentistry, Alexandria, Egypt.

2. Professor of Pediatric Dentistry, Department of Pediatric Dentistry and Dental Public Health, Faculty of Dentistry, Alexandria University, Alexandria, Egypt.

3. Professor of Dental Biomaterials, Department of Dental Biomaterials, Faculty of Dentistry, Alexandria University, Alexandria, Egypt.

4. Associate Professor of Pediatric Dentistry, Department of Pediatric Dentistry and Dental Public Health, Faculty of Dentistry, Alexandria University, Alexandria, Egypt.

\section{*Corresponding author:}

E-mail:dr.nadanabil90@gmail.com

\section{INTRODUCTION}

Prevention, alongside with, arresting caries are the main goals in pediatric dental treatment. Silver diamine fluoride is well documented for its important role in the dental setting and has been included in the caries management protocols by AAPD (1). In 2014, SDF has been approved as dentinal sensitivity treatment by the US Food and Drug Administration (2).

Silver diamine fluoride (SDF) has shown effectiveness in both hardening of tooth structure and killing bacteria. Therefore, it can be used for both prevention and arrest of dental caries (3). In vitro studies $(4,5)$ demonstrated that SDF, reduces dentin demineralization,(5) enhances tooth remineralization, increases the $\mathrm{pH}$ of biofilm, (6) and has antimicrobial effect against cariogenic bacteria(4). Mei et al. (2014) (6) studied extracted primary teeth with cavities of children who received biannual applications of SDF using micro-computed tomography, scanning electron microscope. The arrested lesions were remineralized by calcium and phosphate ions and had intact collagen fibers. It was suggested that the SDF provides alkaline environment that promotes the formation of covalent bond between phosphate ions from saliva and the intact collagen which becomes binding site for calcium ions which leads to apatite nucleation through the collagen (6).

Horst et al. (2016) (7) in a literature review showed that SDF has been suggested for patients with high caries risk, medically compromised, or for those with special needs. Moreover, it has been used to treat patients with no access to dental care, uncooperative or very young patients who require multiple treatment visits until the child grows older and restorations can be performed on the SDF-treated teeth.

However, SDF has shown some limitations. Esthetics for example; due to black staining of the tooth surface as a result of silver precipitate was of great concern for children and their parents. (8) Yasmi et al.(8) (2019) who investigated parental acceptance of SDF, concluded that most parents had concerns about the esthetic impact of SDF. Other adverse effects of SDF treatment were chewing difficulties, food accumulation and difficulty to maintain proper oral hygiene(9). Consequently, two alternative ways has been 
Soliman et al.

suggested to overcome this problem. One of them is the addition of saturated potassium iodide (KI) solution before SDF treatment to react with free silver ions and form a creamy white colored product that is silver iodide (10). However, Koizumi et al. (2016) (11) found that dentin pretreatment with silver diamine fluoride-potassium iodide adversely affected adhesion of resin-based adhesives and RMGIC to dentin. The other alternative is the application of composite or GIC restorations after the application of SDF to mask the black color of the carious lesion to achieve better esthetics and improve chewing ability (12). This technique is called Silver Modified Atraumatic Restorative Technique (SMART).(13)

Wu et al. (2016) (14) showed that the bond strengths of composite to sound primary molars was insignificantly affected by the pretreatment of the primary dentin with 38\% SDF. Ng et al (2020) (15) demonstrated that SDF treatment of artificial carious dentin in permanent teeth has no significant effect on bond strength of GIC to demineralized dentin. Moreover, Uzet et al (2013) revealed that pretreatment of sound permanent molar with 38\% SDF did not affect the microleakage between tooth structure and composite resin.(16) However, there is little information about the effect of $38 \%$ SDF dentin pretreatment on adhesive interface between RMGIC cement and primary dentin. So, the aim of this study was to evaluate microleakage and shear bond strength of RMGIC to primary dentin after SDF pretreatment. Thus, the null hypothesis is that primary dentin pretreatment with or without $38 \%$ SDF yields similar effect on the microleakage and shear bond strength of RMGIC to primary dentin.

\section{MATERIALS AND METHODS}

This study was done after approval of research ethic committee (0036-6/2019) in Faculty of Dentistry, Alexandria University.

Sample size estimation: Based on a previous study by Puwanawiroj et al. in (2018) (17) adopting a power of $80 \%$ and a level of significance $95 \%$, the minimal required sample size was found to be 20 teeth per group (number of groups=4) with total $=80$ teeth. It was calculated using $G$ power 3.1.9.2 (18).

Study sample: Eighty freshly extracted human primary molars were collected from the out-patient clinics of the Faculty of Dentistry, Alexandria University, Ministry of Health Hospital clinics and from private clinics.

Tooth inclusion and exclusion criteria: Sound primary molars with no restorations, cracks, enamel defects or developmental anomalies were only chosen for the study. Teeth were cleaned and stored in distilled water. Teeth were randomly allocated based on evaluation method and type of dentin pre-treatment into four groups $n=20$.The randomization was done using random allocation software program.

Microleakage test: For group I $(n=20)$ and group II $(n=20)$, teeth were sealed apically with sticky wax (Dentsuply International. Milford, USA) and embedded in chemically cured acrylic resin. class V $(3 \mathrm{~mm} \times 2 \mathrm{~mm} \times 1.5 \mathrm{~mm})$ cavities were made in the middle third of the buccal surface of each tooth (19). A carbide bur size \#330 (SS White Bure, New Jersey) with high-speed hand-piece was used under water coolant. The depth of the cavity was adjusted to $1.5 \mathrm{~mm}$ by inserting full length of the bur. Bevels were not made at the
Effect of SDF on adhesion of RMGIC to primary dentin. enamel margins of the cavities. Each 10 cavities the bur was changed with new one to avoid dullness (20).

Teeth were randomly assigned into two groups. Group I (experimental group): cavities were treated with 38\% SDF solution (Elevate oral care, USA) using a micro-brush for three minutes, then rinsed with water for 30-second. (17) Group II (control group): cavities were only treated with distilled water for three minute then rinsed for 30 seconds. (17) For both groups, cavity conditioner (GC America Inc., Alsip, Ill., USA) was applied for 10 seconds using a cotton pellet then rinsed with water. Cavities were restored with RMGIC (Fuji II LC, GC, Tokyo, Japan) then light cured for 20 seconds using LED light curing system (Ivoclar Vivadent, Germany). All steps were done according to manufacturer instructions (21). After that specimen were stored in distilled water at $37^{\circ} \mathrm{C}$ for 24 hours

Teeth were thermo-cycled for 500 cycles between 5 and $55^{\circ} \mathrm{C}, 30$ seconds of immersion in each water bath, and 30 seconds of transfer time between conditioning. After thermocycling, each tooth was covered with a two layers of nail varnish except for $1 \mathrm{~mm}$ around the restorative margins. Teeth were then immersed in 1\% methylene blue for $24 \mathrm{~h}$. After 24h, the superficial dye on the teeth was removed through washing it by slurry of pumice. Then the teeth were sectioned longitudinally in bucco-lingual direction using a diamond saw under water coolant (22). (Isomet 4000, microsaw Buehler, USA).

Calibration was done by examining another 20 specimens with one week interval. The kappa statistics was used to determine intra-examiner reliability. Weighted kappa statistics revealed a significant very good agreement between two assessments of microleakage scores at two different times in both groups as $\mathrm{K}(70 \% \mathrm{CI})=0.703$. This indicates consistency of the examiner in the application of scoring criteria over time. Evaluation was done by one examiner (demonstrator at Dental Biomaterials Department, Alexandria University).

Stereomicroscope (Olympus SZ1145, Olympus Optical Co., LTD. Tokyo, Japan) at x40 magnification was used to assess the dye penetration degree in the occlusal and gingival walls. The score at the cervical and occlusal margins were recorded at each tooth section and the highest score was chosen to represent the microleakage for the tooth section. Then, the greatest score of both two sections of each tooth was recorded to represent the overall microleakage of the whole tooth to simplify the results. Microleakage was recorded according to the criteria suggested by Silveira de Araïjo. Score $0=$ no leakage, $1=$ leakage extending to half the depth of cavity, 2=leakage extending to more than half of the depth of cavity and $3=$ leakage extending to the cavity floor (Table1) (22).

Shear bond strength test: (17) The roots of extracted primary molars for group III $(n=20)$ and group IV $(n=20)$ were cut off $2 \mathrm{~mm}$ below the cementum-enamel junction using highspeed diamond under water coolant. The specimens were mounted in acrylic resin molds perpendicular to the long axis of it with their buccal surface displayed. Convex buccal surfaces of the crowns were gently mechanically ground using a low-speed diamond-disk with water coolant to obtain flat dentin surface. Group III (experimental) was treated with 38\% SDF solution for 3 minutes using micro-brush, followed by a 30seconds rinse with water. Group IV (Control) teeth were 
Soliman et al.

treated with distilled water for 3 minutes then rinsed for 30 seconds. Then cavity conditioner was applied to flat dentin of both groups for 10 seconds then washed and dried gently with compressed air. To create a standardized bonding area a plastic cylindrical shaped mold with internal diameter of $3 \mathrm{~mm}$ and height of $2 \mathrm{~mm}$ was placed at right angle on the cut surface of the polished flat dentin and restored with RMGIC (Fuji II LC, GC, Tokyo, Japan) in increments then each one was light cured for 20 seconds $(17,23)$.

Universal Testing machine (AUTOGRAPH, AG5KNIS MS, SHIMADZU Co. Kyoto, Japan) was used to assess the shear bond strength. Shear load was directed parallel to the bonded interface with a sharp blade at cross head speed of $1 \mathrm{~mm}$ per minute until failure occurred. The following equation was used to calculate the shear bond strength:

Shear bond strength in Megapascals $(\mathrm{MPa})=$ the maximum failure load in Newtons (N) / surface area of the bonded interface $\left(\mathrm{mm}^{2}\right)$ (23).

Failure mode assessment.(24) Failure modes was evaluated by a single examiner (demonstrator at Dental Biomaterial Department, Alexandria University) using stereomicroscope at $\mathrm{x} 40$ magnification, and classified as: Adhesive (failure at the interface of RMGIC and the dentin surface), Cohesive (failure within the dentin or RMGIC) and Mixed (combination of adhesive and cohesive failure).

Statistical analysis:

Normality was checked using descriptive statistics, plots (histogram and box plot and Shapiro Wilk test. Median, inter quartile range (IQR) and percentages represented the microleakage scores. Micro-leakage scores between study groups were compared using Mann Whitney U test. Shear bond strength (SBS) was presented using mean and standard deviation while frequencies and percentages were used to present the mode of failure. Independent t test was used to assess differences in SBS between study groups. Mode of failure was analyzed using Chi square test. Level of significance was set at $p \leq 0.05$. IBM SPSS statistical software (version 25) was used to analyze the data.

Table 1: Dye penetration scores

\begin{tabular}{|c|l|}
\hline Score & \multicolumn{1}{|c|}{ Criteria } \\
\hline 0 & No leakage \\
\hline 1 & Leakage extending to half the depth of cavity \\
\hline 2 & $\begin{array}{l}\text { Leakage extending to more than half of the depth of } \\
\text { cavity }\end{array}$ \\
\hline 3 & Leakage extending to the cavity floor \\
\hline
\end{tabular}

\section{RESULTS}

Microleakage evaluation: Table 2 shows a comparison between overall microleakage scores among group I (SDF pretreated primary dentin) and group II (control) at both occlusal and cervical margin.

Results for group I (primary dentin pretreated with 38\% SDF), showed one specimen (5\%) with score 0 denoting no microleakage, 17 (85\%) specimens with score 1, one specimen (5\%) with score 2 and one specimen (5\%) with score 3. (Figure 1) For group II (no pretreatment with SDF), 3 (15\%) specimens showed score $0,8(40 \%)$ specimens showed score 1, 6 (30\%) specimens showed score 2 and $3(15 \%)$ specimens showed score 3. (Figure 2) Microleakage scores in the
Effect of SDF on adhesion of RMGIC to primary dentin. experimental group treated with SDF were less than the control group. Most specimens in the SDF pretreated group scored 1, while the number of teeth scored 2 and 3 in the control group were more than that in the experimental group. However, the difference in the median overall microleakage scores between the SDF pretreated group and the control group was not statistically significant using Mann Whitney U test $(p=0.21)$. (Table 2$)$

Shear bond strength (SBS) evaluation represented a mean of 8.28 $\mathrm{MPa}$ in the experimental group and $4.40 \mathrm{MPa}$ in the control group. Descriptive statistics of the shear bond strength (MPa) of the experimental and control groups are illustrated in (Figure 3). The highest bond strength value was recorded in the SDF pretreated group while the lowest shear bond strength value was recorded in the control group. Using independent $t$ test, 38\%SDF pretreated primary dentin presented statistically significant higher bond strength $(\mathrm{P}=<0.0001)$.

Failure mode assessment results: Most of failures were classified as mixed mode (85\%) in group III (38\% SDF pretreated) and adhesive mode (80\%) in group IV (control group). Only one tooth in the SDF pretreated group showed cohesive mode of failure which was absent in the control group. Using Chi square test, the failure mode assessment showed statistically significant difference between both groups, in favor of the $38 \%$ SDF pretreated group ; as the mixed mode of failure represents stronger bond between RMGIC and primary dentin that the adhesive mode of failure. $(\mathrm{P}<0.0001)$. (Figure 4)

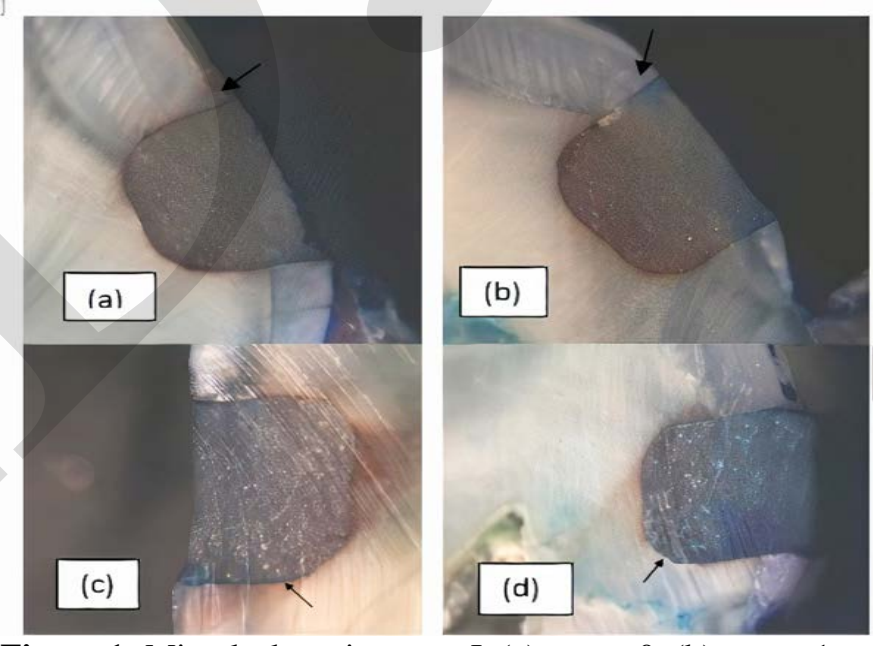

Figure 1: Microleakage in group I. (a): score 0, (b): score 1, (c): score 2, (d): score 3
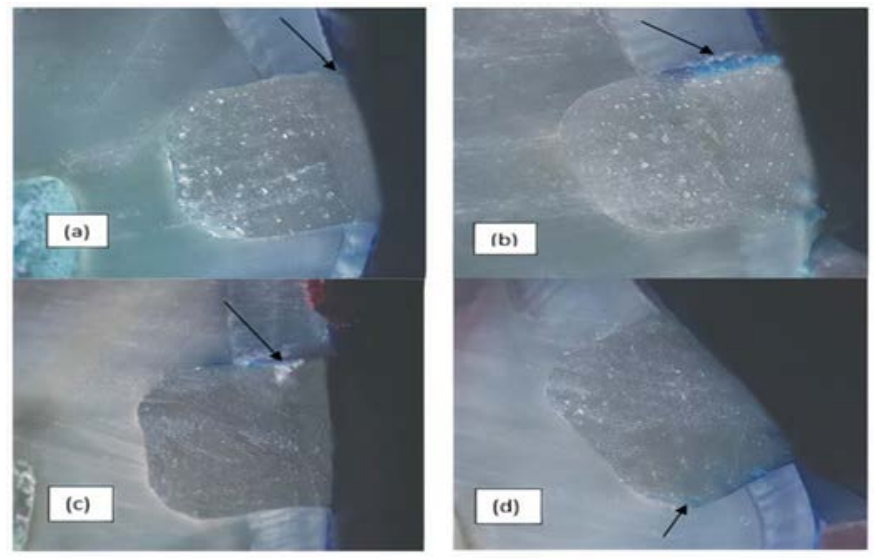
Soliman et al.

Figure 2: Showing microleakage in group II. (a): score 0, (b): score 1 , (c): score 2 , (d): score 3

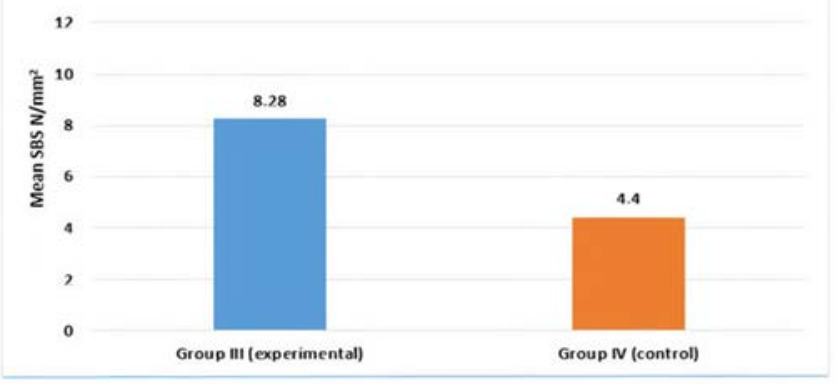

Figure 3: Mean shear bond strength values among the study groups

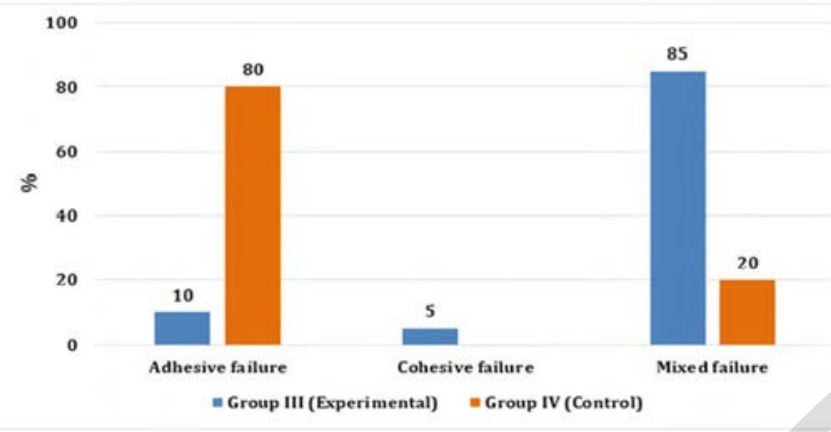

Figure 4: Mode of failure between the study groups

Table 2: The overall microleakage scores in group I and group II

\begin{tabular}{|c|c|c|}
\hline Microleakage score & $\begin{array}{c}\text { Group I (Test) } \\
\mathbf{n = 2 0}\end{array}$ & $\begin{array}{c}\text { Group II(Control) } \\
\mathbf{n = 2 0}\end{array}$ \\
\cline { 2 - 3 } & \multicolumn{2}{|c|}{$\mathbf{n ( \% )}$} \\
\hline Score 0 & $1(5 \%)$ & $3(15 \%)$ \\
\hline Score 1 & $17(85 \%)$ & $8(40 \%)$ \\
\hline Score 2 & $1(5 \%)$ & $6(30 \%)$ \\
\hline Score 3 & $1(5 \%)$ & $3(15 \%)$ \\
\hline Median & 1.00 & 1.00 \\
\hline Inter quartile range & $(1-1)$ & $(1-2)$ \\
\hline (Min-Max) & $(0-3)$ & $(1-3)$ \\
\hline P value & \multicolumn{2}{|c|}{$0.21 *$} \\
\hline
\end{tabular}

\section{DISCUSSION}

In the present study there was no significant difference in the microleakage between teeth treated with SDF and the untreated teeth. However, there was a significant increase in the shear bond strength of the SDF treated primary dentine.

Microleakage was tested as it is widely accepted and preferred method. It is readily available, cheap and non-toxic.(16) Moreover, nano-leakage describes the mechanism of dye penetration in the hybrid layer in dentin not the gap between restoration and the cavity margin.(25)

Proper seal of the cavity and having a good bond to the tooth structure are main factors of the restoration to prevent secondary caries. (26)Therefore, evaluation of the sealing ability and bond strength of the restorations is important to assess its performance.
Effect of SDF on adhesion of RMGIC to primary dentin.

There are different commercially available concentrations of SDF used in dentistry 12\%, 30\% and 38\%. The concentration of $38 \%$ was chosen for the present study according to Fung et al. (27) (2018) who concluded that 38\% SDF was statistically significant in caries prevention and arrest in primary teeth in comparison to $12 \%$ SDF.

The main problem of Class $\mathrm{V}$ cavities is the leakage at the cervical margin which is a moisture sensitive area. Glass ionomer restoration is used in these areas that cannot be isolated which makes it the material for choice in class $\mathrm{V}$ cavities. (28) According to Stewardson et al. (2012)(29)class $\mathrm{V}$ cavities restored with glass ionomer had more survival rate than using any other restorative material. That is why class $\mathrm{V}$ cavity was chosen to test microleakage of RMGIC in the study.

Results of the present study showed that there was no significant difference between 38\%SDF pretreated primary dentin group and the control group as regard to the microleakage. This was in line with Gupta et al. (30) (2019) who reported that silver diamine fluoride -potassium iodide (SDF-KI) pretreated teeth did not have a significant difference in microleakage between RMGIC and tooth structure when compared to other groups in the study which were treated with chlorhexidine and distilled water. Silver diamine fluoride solution was applied to dentine surface, and then KI using a standardized micro brush. The reaction products were washed off with copious distilled water and then blot dried. These results are also supported by Uzel et al. (16) (2013)who found no significant difference in microleakage between the group of permanent teeth treated with 38\% SDF before the application of resin composite and the group of teeth with no treatment. Moreover, in the present study, it was noticed that dye leakage of $70 \%$ of specimens scored 1 in the SDF pretreated group were in enamel without reaching the dentin interface. This could be attributed to the dentinal tubules occluded by proteins precipitate formed by silver ions (31). Moreover, the reaction of fluoride ions with calcium ions forms a precipitate of calcium fluoride $\left(\mathrm{CaF}_{2}\right)$ plugging dentinal tubules (32).

According to the results of this study, it was found that pretreatment of sound primary dentin with SDF raised significantly SBS between RMGIC and the primary dentin. Mixed type of failure was predominant in the test group while adhesive type of failure was predominant in the control group and the difference between the two groups was significant. The observed mode of failure indicates that the retentive strength between SDF pretreated primary dentin and RMGIC was higher than that in the control group(33). Silver phosphate $\left(\mathrm{Ag}_{3} \mathrm{PO}_{4}\right)$ and calcium fluoride $\left(\mathrm{CaF}_{2}\right)$ are formed by the reaction of SDF with calcium hydroxyapatite (34). Silver phosphate may react with the carboxylic group of RMGIC increasing the bond between it and primary dentin (30). Moreover, Fixation of the organic content leads to contraction of the organic substance inside dentinal tubules increasing interlocking between dentinal tubules which may also be a cause of the increased bond strength (30). According to Frohlich et al. (35) (2019) in a systematic review, the adhesion of glass ionomer cement (GIC) restorations to tooth structure is not jeopardized by SDF pretreatment of primary dentin, on the contrary it may improve it. This may be attributed to the fact that GIC adheres chemically to tooth structure. The precipitate of silver deposits and silver ions resulted from SDF pretreatment could improve the ionic bond to GIC. 
Soliman et al.

Moreover, light curing of the RMGIC for 20 seconds may have an effect on the increase of the shear bond strength between SDF pretreated primary dentin and RMGIC. Wang et al. (36) who found that demineralized dentin treated with SDF then light cured had higher SBS than demineralized dentin treated with SDF without light curing or not treated at all. It was revealed that dentin surface darkened more when SDF was light cured which indicates more precipitation of more metallic silver increasing the ionic interaction between GIC and dentin (36).

The results of this study were in line with Gupta et al (30) who concluded that SDF-KI significantly raised SBS of RMGIC to sound dentin although they added KI on the SDF before the application of RMGIC to mask the black color of SDF.

The present study showed some limitations. Being invitro, it does not totally represent the intra-oral environment like changing ph. In the microleakage test, it was hard to distinguish the dark blue color of the methylene blue dye from the black stain caused by SDF pretreatment of tooth structure The results of this study revealed that SDF can be used without compromising the bond strength between RMGIC and sound primary dentin. The good bond between the restoration and tooth structure achieves a good seal besides the antibacterial property of the SDF which helps in the prevention of secondary caries with the limitation of the grey discoloration of RMGIC. According to these results, the null hypothesis was partially rejected. However, future studies are needed to test its biological effect on the pulp and its interaction with other restorative materials like composite as well as its clinical performance. Moreover, it is recommended to study whether light cured or chemical cured GIC have different effects on micro-leakage and shear bond strength.

\section{CONCLUSION}

According to the results of this study, it was concluded that $38 \%$ SDF pretreatment of primary dentin did not have an adverse effect on the marginal seal. However, SBS of pretreated primary dentin was significantly improved.

\section{CONFLICT OF INTEREST}

The authors declared that there was no conflict of interest.

The authors received no specific funding for this work.

\section{REFERENCES:}

1. American Academy of Pediatric Dentistry. Caries-risk assessment and management for infants, children, and adolescents 2020 [cited 2020 july 9,]. Available from: https://www.aapd.org/globalassets/media/policies_guidel ines/bp_cariesriskassessment.pdf.

2. U.S. Food and Drug Administration. 510(k) Premarket Notification 2014 [updated 2019July 10, 2020]. Available from: https://www.accessdata.fda.gov/scripts/cdrh/cfdocs/cfpm n/pmn.cfm?ID=K102973.

3. Crystal YO, Niederman R. Silver diamine fluoride treatment considerations in children's caries management. Pediatr Dent. 2016;38:466-71.

4. Chu CH, Mei L, Seneviratne CJ, Lo EC. Effects of silver diamine fluoride on dentine carious lesions induced by Streptococcus mutans and Actinomyces naeslundii biofilms. Int J Paediatr Dent. 2012;22:2-10.
Effect of SDF on adhesion of RMGIC to primary dentin.

5. Mei ML, Ito L, Cao $\mathrm{Y}$, Li QL, Lo EC, Chu CH. Inhibitory effect of silver diamine fluoride on dentine demineralisation and collagen degradation. $\mathrm{J}$ Dent. 2013;41:809-17.

6. Mei ML, Ito L, Cao Y, Lo ECM, Li QL, Chu CH. An ex vivo study of arrested primary teeth caries with silver diamine fluoride therapy. J Dent. 2014;42:395-402.

7. Horst JA, Ellenikiotis H, Milgrom PL. UCSF protocol for caries arrest using silver diamine fluoride: rationale, indications and consent. J Claif Dent Assoc. 2016;44:1628.

8. Yasmi CO, Bianca K, Victoria RH. Parental expressed concerns about silver diamine fluoride (sdf) treatment. J Clin Pediatr Dent. 2019;43:155-60.

9. Jiang M, Mei ML, Wong MCM, Chu CH, Lo ECM. Effect of silver diamine fluoride solution application on the bond strength of dentine to adhesives and to glass ionomer cements: a systematic review. BMC oral health. 2020;20:40-9.

10. Zhao IS, Mei ML, Burrow MF, Lo EC-M, Chu C-H. Effect of silver diamine fluoride and potassium iodide treatment on secondary caries prevention and tooth discolouration in cervical glass ionomer cement restoration. Int J Mol Sci. 2017;18:340-51.

11. Koizumi H, Hamama HH, Burrow MF. Effect of a silver diamine fluoride and potassium iodide-based desensitizing and cavity cleaning agent on bond strength to dentine. Int J Adhes Adhes. 2016;68:54-61.

12. Zhao IS, Chu S, Yu OY, Mei ML, Chu CH, Lo ECM. Effect of silver diamine fluoride and potassium iodide on shear bond strength of glass ionomer cements to cariesaffected dentine. Int Dent J. 2019;69:341-7.

13. Sarvas EW. Medical management of dental caries. In: Nelson TM, Webb JR, editors. Dental Care for Children with Special Needs: A Clinical Guide. 1st ed. Cham: Springer International Publishing; 2019:195-214.

14. Wu DI, Velamakanni S, Denisson J, Yaman P, Boynton JR, Papagerakis P. Effect of silver diamine fluoride (sdf) application on microtensile bonding strength of dentin in primary teeth. Pediatr Dent. 2016;38:148-53.

15. Ng E, Saini S, Schulze KA, Horst J, Le T, Habelitz S. Shear bond strength of glass ionomer cement to silver diamine fluoride-treated artificial dentinal caries. Pediatr Dent. 2020;42:221-5.

16. Uzel I, Ulukent O, Cogulu D. The effect of silver diamine fluoride on microleakage of resin composite. J Int Dent. 2013;6:105-8.

17. Puwanawiroj A, Trairatvorakul C, Dasanayake AP, Auychai P. Microtensile bond strength between glass ionomer cement and silver diamine fluoride-treated carious primary dentin. Pediatr Dent. 2018;40:291-5.

18. Faul F, Erdfelder E, Lang A-G, Buchner A. G*Power 3: A flexible statistical power analysis program for the social, behavioral, and biomedical sciences. Behav Res Methods. 2007;39:175-91.

19. El-Ashiry EA, Bakry NS, Farsi N, Farsi D. Microleakage evaluation of two different nano-restorative materials in primary molars: In vitro study. Life Sci J. 2012;9:2292300.

20. Ameri H, Chasteen JE, Ghavamnasiri M, Maghami A. Effect of load cycling on nanoleakage of butt joint and 
Soliman et al.

beveled occlusal enamel margins in class $\mathrm{V}$ composite resin restorations. Arch Oral Res. 2010;6:231-7.

21. GC CORPORATION Fuji II LC CAPSULE Instructions For Use. GC CORPORATION Tokyo, Japanhttps://www.manualslib.com/manual/1497627/GcCorporation-Fuji-Ii-Lc-Capsule.html\#manual [April 13, 2020].

22. Silveira de Araujo C, Incerti da Silva T, Ogliari FA, Meireles SS, Piva E, Demarco FF. Microleakage of seven adhesive systems in enamel and dentin. J Contemp Dent Pract. 2006;7:26-33.

23. Poggio C, Beltrami R, Scribante A, Colombo M, Lombardini M. Effects of dentin surface treatments on shear bond strength of glass-ionomer cements. Ann stomal (Roma).2014; 5:15-22.

24. Chai $Y$, Lin $H$, Zheng G, Zhang $X$, Niu G, Du Q. Evaluation of the micro-shear bond strength of four adhesive systems to dentin with and without adhesive area limitation. Biomed Mater Eng. 2015;26 :63-72.
Effect of SDF on adhesion of RMGIC to primary dentin.

25. Makishi P, André C, Ayres A, Martins A, Giannini M. Effect of storage time on bond strength and nanoleakage expression of universal adhesives bonded to dentin and etched enamel. Oper Dent. 2016;41:305-17.

26. Zhang N, Melo MAS, Chen C, Liu J, Weir MD, Bai Y, et al. Development of a multifunctional adhesive system for prevention of root caries and secondary caries. Den Mater. 2015;31:1119-31.

27. Fung MHT, Duangthip D, Wong MCM, Lo ECM, Chu $\mathrm{CH}$. Randomized clinical trial of $12 \%$ and $38 \%$ silver diamine fluoride treatment. J Dental Res. 2018;97:171-8.

28. Gupta SK, Gupta J, Saraswathi V, Ballal V, Acharya SR. Comparative evaluation of microleakage in Class $\mathrm{V}$ cavities using various glass ionomer cements: An in vitro study. J Interdiscip Dent. 2012;2:164-9. 